\title{
Case Report \\ Primary Peritonitis: An Index Case of Mycoplasma hominis Infection in a Healthy Female
}

\author{
Sabrina Drexel (iD) and Daniel Tseng ${ }^{2}$ \\ ${ }^{1}$ Oregon Health and Science University, 3181 SW Sam Jackson Park Rd., Portland, OR 97239, USA \\ ${ }^{2}$ Legacy Good Samaritan Hospital, 1015 NW 22nd Ave., Portland, OR 97210, USA \\ Correspondence should be addressed to Sabrina Drexel; drexel@ohsu.edu
}

Received 10 October 2017; Accepted 8 January 2018; Published 6 February 2018

Academic Editor: Alexander R. Novotny

Copyright (C) 2018 Sabrina Drexel and Daniel Tseng. This is an open access article distributed under the Creative Commons Attribution License, which permits unrestricted use, distribution, and reproduction in any medium, provided the original work is properly cited.

\begin{abstract}
Introduction. Primary peritonitis in healthy immunocompetent individuals is rare. Several case reports of Streptococcus species causing peritonitis have been described. Here, we present the first case of Mycoplasma hominis as the cause of primary peritonitis in a healthy woman. Case Report. A 42-year-old female with history of uterine fibroids was admitted with abdominal pain and intraperitoneal fluid of unknown etiology. She was initially managed nonoperatively and empirically treated with broad spectrum antibiotics. Blood and urine cultures were unrevealing. Increasing abdominal pain and peritoneal fluid prompted diagnostic laparoscopy which revealed a dense fibrinous exudate covering the entire peritoneal cavity. Peritoneal fluid and biopsies were sent for cytology and culture. The peritoneal fluid was eventually sent for $16 \mathrm{~s}$ ribosomal analysis, which discovered Mycoplasma hominis RNA. Her antibiotics were narrowed, and she eventually made a full recovery. Discussion. M. hominis is a rare source of systemic infection but has been known to colonize the urogenital tract and cause localized infections. This is the first presentation of $M$. hominis causing primary peritonitis in a healthy immunocompetent female. Multidisciplinary management of these patients is critical to achieve a timely diagnosis. Surgical exploration is often unavoidable to rule out secondary peritonitis.
\end{abstract}

\section{Introduction}

Peritonitis is a clinical syndrome that is derived from peritoneal irritation. This diagnosis may be suspected by abdominal exam findings such as voluntary and involuntary guarding, localized tenderness, and rebound tenderness. Peritonitis may occur as a primary process seen in patients with severe liver disease, immunocompromised states, nephrotic syndrome, or heart disease. However, surgeons often are involved in the diagnosis and management of peritonitis secondary to complications involving visceral organs. These patients typically require surgical intervention. In contrast, primary peritonitis is often diagnosed with paracentesis and treated with antibiotics. The most common organisms that cause primary peritonitis are gram-negative enteric bacteria (60\%), including Escherichia coli and Klebsiella pneumoniae. Gram-positive cocci account for 25\%, in which Streptococcus is most commonly isolated [1].
Primary peritonitis is an extremely rare entity in healthy adults. There are numerous case reports of Group A Streptococcus (GAS) and Streptococcus pneumoniae causing primary peritonitis in young previously healthy individuals [1-3]. Primary peritonitis with Mycoplasma hominis has been described in the renal transplant population [4-6]; however, there is no known reported case of primary peritonitis caused by Mycoplasma hominis in a healthy nonimmunocompromised adult. To our knowledge, we present the first case of Mycoplasma hominis peritonitis in an otherwise healthy young female.

\section{Case Report}

A 42-year-old female was seen in the emergency department with three days of severe abdominal pain and sepsis. Medical history included menometrorrhagia due to uterine fibroids and previous endometrial ablation in 2015. 


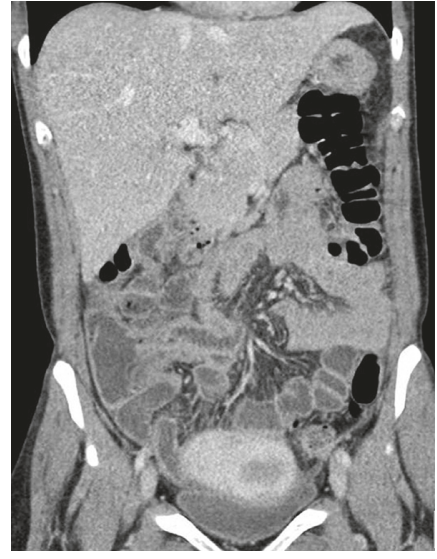

(a)

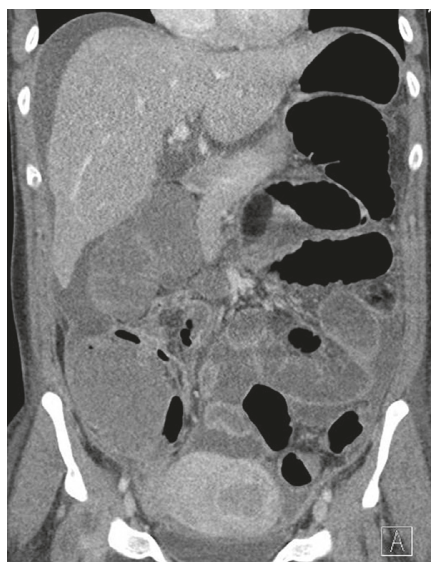

(c)

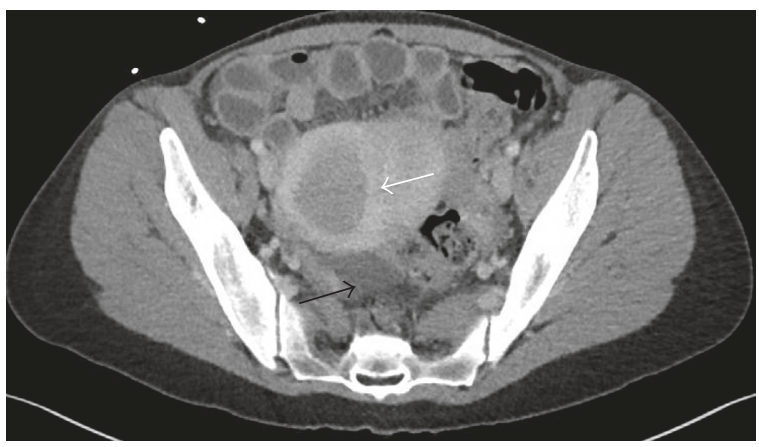

(b)

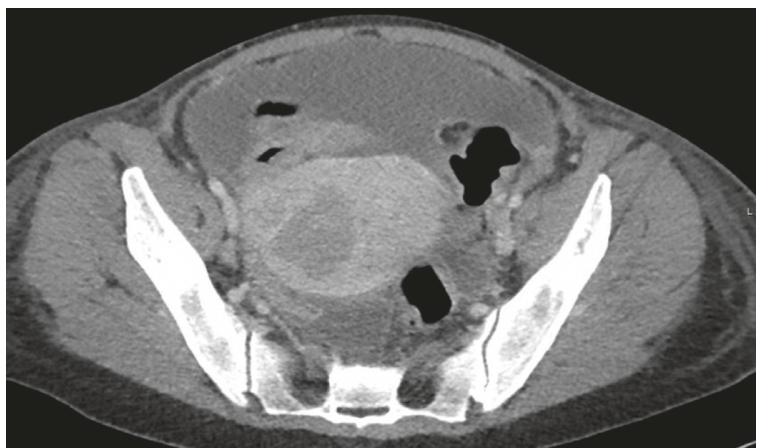

(d)

FIGURE 1: Initial CT scan upon presentation (a, b) with uterine fibroids (white arrow) and minimal free fluid (black arrow). CT scan four days after admission with worsening ascites $(c, d)$.

She used a contraceptive vaginal ring for three months prior and Provera for 2 days prior to presentation for persistent vaginal bleeding. Physical examination revealed the following vital signs: temperature $39.2^{\circ} \mathrm{C}$; blood pressure $136 / 78 \mathrm{mmHg}$, pulse 132 beats/min, and respiratory rate 16 breaths/min. Oxygen saturation was $100 \%$ on room air. Physical exam revealed tenderness throughout the lower abdomen with voluntary guarding. Labarotory data revealed WBC of $12,000 / \mu \mathrm{L}, \mathrm{Hgb}$ of $7.8 \mathrm{gm} / \mathrm{dL}$ and $\mathrm{Hct}$ of 25.7, sodium of $127 \mathrm{mmol} / \mathrm{L}$, and chloride of $96 \mathrm{mmol} / \mathrm{L}$. Lactate was normal at $1.5 \mathrm{mmol} / \mathrm{L}$. Initial CT scan showed uterine masses, consistent with known fibroids, and minimal pelvic fluid (Figure 1).

She was initially admitted to the medical service for sepsis. Given her lower abdominal pain, Gynecology was consulted for presumed pelvic inflammatory disease (PID). She was started on empiric antibiotics for PID (ceftriaxone IV $1 \mathrm{~g}$ every 24 hours, azithromycin IV $500 \mathrm{mg}$ every 24 hours, and metronidazole IV $500 \mathrm{mg}$ every 8 hours). Blood, urine, and stool cultures were sent upon admission, all of which were negative.

Her pain continued to progress with rising leukocytosis to $20,000 / \mu \mathrm{L}$. A repeat CT scan on hospital day four showed interval development of ascites (Figure 1). Surgical consultation was obtained, and she was found to have an acute abdomen. She was taken for a diagnostic laparoscopy, which revealed a dense fibrinous exudate and significant ascites in all four quadrants of the abdomen (Figure 2). Multiple peritoneal biopsies were taken, and peritoneal fluid was sent for gram stain, cultures, and cytology.

Postoperatively, carcinoembryonic antigen (CEA) and CA-125 levels were obtained. CEA was within the normal range, and CA-125 was mildly elevated at $56.8 \mathrm{U} / \mathrm{mL}$ (normal $0-35.0 \mathrm{U} / \mathrm{mL}$ ). Her WBC fluctuated between 15,000 and $25,000 / \mu \mathrm{L}$. The peritoneal fluid gram stain did not reveal any bacteria. Cytology revealed rare mesothelial cells and abundant neutrophils but no malignant cells. Final pathology report stated the presence of benign fibromembranous tissue with severe acute inflammation and extensive necrosis, consistent with "severe necrotizing acute peritonitis."

Given the extensive inflammatory reaction, infectious disease was consulted. Group A Strep (GAS) peritonitis was suspected, and recommendations were made to continue ceftriaxone at $1 \mathrm{~g}$ IV every 24 hours in addition to one dose of clindamycin $600 \mathrm{mg}$ IV. Azithromycin was added to ceftriaxone three days later (IV $500 \mathrm{mg}$ every 24 hours). A streptolysin $\mathrm{O}$ antibody (ASO) titer was sent but came back 


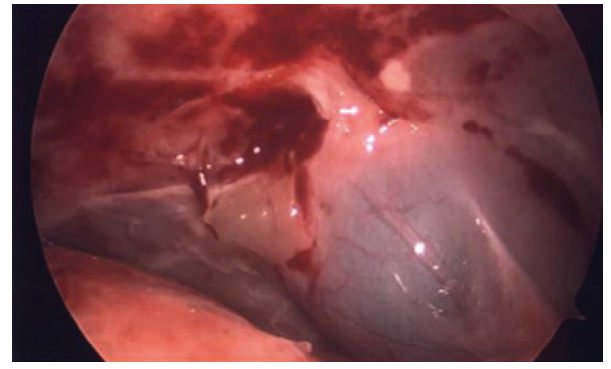

(a)

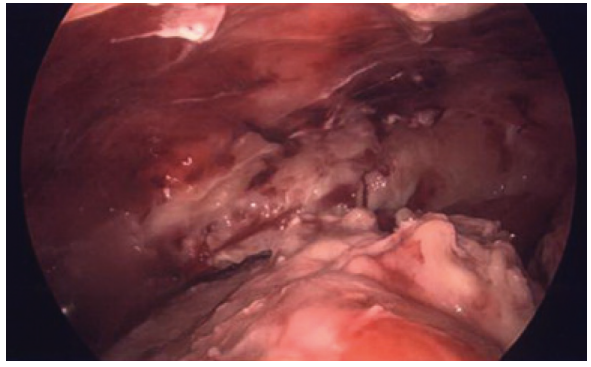

(b)

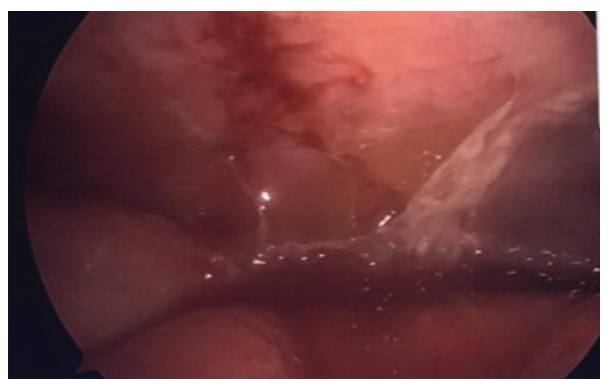

(c)

Figure 2: Diagnostic laparoscopy findings: inflamed peritoneum with densely adhered gallbladder to liver (a); fibrinous exudate throughout the peritoneal cavity (b); gelatinous ascites (c).

normal at $61 \mathrm{I} \mathrm{U} / \mathrm{mL}$ (normal range $0-330 \mathrm{IU} / \mathrm{mL}$ ), arguing against GAS peritonitis. Ceftriaxone was stopped, and azithromycin was transitioned to oral $500 \mathrm{mg}$ daily.

She continued to have persistent abdominal pain and anorexia. A repeat CT scan obtained nine days after surgery (Figure 3) showed peritoneal enhancement and several fluid collections. A diagnostic paracentesis was performed, and the peritoneal fluid was sent to an outside institution for $16 \mathrm{~S}$ ribosome analysis, to isolate bacterial RNA.

Her abdominal pain and leukocytosis slowly improved. She continued to have severe anorexia and food aversion, despite reassuring physical exam findings. On hospital day 23, she was discharged home on oral azithromycin. Several days after discharge, $16 \mathrm{~S}$ ribosome testing revealed Mycoplasma hominis RNA within the peritoneal fluid. Her outpatient antibiotic was changed to oral doxycycline $100 \mathrm{mg}$ twice daily for three months. She was closely followed as an outpatient and gradually demonstrated clinical improvement. She remains well ten months after hospitalization.

\section{Discussion}

This is the first known case of Mycoplasma hominis primary peritonitis in an otherwise healthy female. In our case, detection of this organism proved to be extremely difficult. Standard analysis of blood, peritoneal fluid, and peritoneal biopsy cultures was unable to detect this organism. In contrast, other recognized causes of primary peritonitis in healthy women, such as Streptococcus pneumoniae and Group A Streptococcus, typically have positive cultures, making organism identification more straightforward [1-3].

Streptococcus pneumoniae has been described as a cause of primary peritonitis, especially in the pediatric population $[3,7,8]$. These patients typically present with an acute abdomen, and surgery is often pursued for presumed secondary peritonitis. A retrospective review of 27 case studies from 1966-1997 showed all patients had either positive blood or peritoneal cultures [9]. The patients were majority female, and the most common source of infection appeared to be the genital tract.

Group A Streptococcus (GAS) typically causes upper respiratory infections, although thirty-eight case reports for GAS primary peritonitis have been cited [2]. Most of these patients were female who presented with fevers and significant abdominal pain. All but one patient received exploratory surgery due to concern for secondary peritonitis, even despite negative imaging. $83 \%$ of these patients had positive blood cultures. In a more recent review of the 21 st century case reports on GAS peritonitis, the authors noted none of these patients had free air on imaging, arguing against perforated viscus as the cause of peritonitis. Again, the majority of patients underwent exploratory surgery (92.1\%). They conclude GAS peritonitis may not be as rare as previously suspected [3].

In general, $M$. hominis is unlikely to cause systemic infection, but it does colonize the urogenital tract and can generate localized infections. Up to $50 \%$ of sexually active women are colonized with $M$. hominis in the cervix or vagina [10]. The Mycoplasma species is extremely small and lacks a peptidoglycan cell wall; therefore, it does not appear on gram stain, making it particularly difficult to identify using traditional techniques. The most reliable test for detection of M. hominis is the $16 \mathrm{~S}$ ribosomal RNA gene by PCR [11], although multiplex real time PCR tests are also being developed [12]. However, these tests are not available at all institutions and often takes days to weeks to obtain results. 


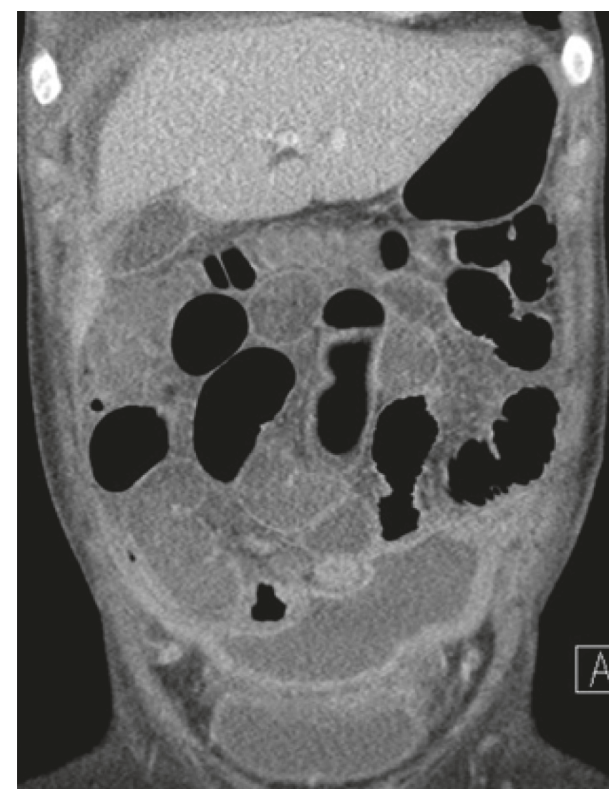

FIGURE 3: CT scan nine days after surgery with persistent ascites and peritoneal enhancement.

The lack of a peptidoglycan cell wall also makes $M$. hominis not susceptible to most broad spectrum antibiotics, including penicillins, cephalosporins, or carbapenems [13]. The treatment of choice is tetracyclines or fluoroquinolones [14].

M. hominis has been known to cause genitourinary inflammatory diseases in adults including pyelonephritis, pelvic inflammatory disease, bacterial vaginosis, and postpartum endometritis. It has a different spectrum of disease in neonates and infants [15]. In this sentinel case, the mode and route of infection is unclear. No vaginal or cervical cultures were taken during her hospitalization to investigate urogenital colonization of M. hominis, but this seems to be the most likely source. It remains unknown whether the patient's contraceptive vaginal ring, large uterine fibroids, and previous myometrial ablations played any role in her increased susceptibility.

In all cases of peritonitis, broad spectrum antibiotics should be started as soon as possible [16]. Surgery often remains the mainstay in diagnosis and treatment of patients with peritonitis. In this situation, primary bacterial peritonitis from a multitude of different organisms may need to be considered. A multidisciplinary approach with consultation of an infectious disease specialist is recommended early in the course of a patient with unclear source of peritonitis. Diligence to ensure there is no other cause for peritonitis is critical to obtain source control, and exploratory surgery is often unavoidable.

\section{Conflicts of Interest}

The authors declare that they have no conflicts of interest.

\section{References}

[1] J. Such and B. A. Runyon, "Spontaneous bacterial peritonitis," Clinical Infectious Diseases, vol. 27, no. 4, pp. 669-674, 1998.
[2] M. Malota, T. W. Felbinger, R. Ruppert, and N. C. Nüssler, "Group A streptococci: a rare and often misdiagnosed cause of spontaneous bacterial peritonitis in adults," International Journal of Surgery Case Reports, vol. 6, pp. 251-255, 2015.

[3] B. Moftakhar, Y. Patel, J. Poblete, and J. Myers, "Primary peritonitis due to Streptococcus pyogenes: report of 2 cases and review of the 21st-century literature," Infectious Diseases in Clinical Practice, 2017. in press.

[4] M. Pastural, V. Audard, M. P. Bralet et al., "Mycoplasma hominis infection in renal transplantation," Nephrology Dialysis Transplantation, vol. 17, no. 3, pp. 495-496, 2002.

[5] P. Rohner, I. Schnyder, B. Ninet et al., "Severe Mycoplasma hominis infections in two renal transplant patients," European Journal of Clinical Microbiology \& Infectious Diseases, vol. 23, no. 3, pp. 203-204, 2004.

[6] B. Camara, M. Mouzin, D. Ribes et al., "Perihepatitis and perinephric abscess due to Mycoplasma hominis in a kidney transplant patient," Experimental and Clinical Transplantation, vol. 5, no. 2, pp. 708-709, 2007.

[7] K. R. Nielsen, T. Ejlertsen, S. El-Batran, and J. Prag, "A fiveyear survey of pneumococcal peritonitis in two Danish counties-incidence, diagnosis and clinical entities," Clinical Microbiology and Infection, vol. 9, no. 7, pp. 738-740, 2003.

[8] D. D. Dugi III, D. M. Musher, J. E. Clarridge III, and R. Kimbrough, "Intraabdominal infection due to Streptococcus pneumoniae," Medicine, vol. 80, no. 4, pp. 236-244, 2001.

[9] C. Hemsley and S. J. Eykyn, "Pneumococcal peritonitis in previously healthy adults: case report and review," Clinical Infectious Diseases, vol. 27, no. 2, pp. 376-379, 1998.

[10] K. B. Waites, L. Xiao, V. Paralanov, R. M. Viscardi, and J. I. Glass, "Molecular methods for the detection of Mycoplasma and Ureaplasma infections in humans: a paper from the 2011 William Beaumont Hospital Symposium on molecular pathology," Journal of Molecular Diagnostics, vol. 14, no. 5, pp. 437-450, 2012.

[11] T. Yoshida, S. Maeda, T. Deguchi, T. Miyazawa, and H. Ishiko, "Rapid detection of Mycoplasma genitalium, Mycoplasma hominis, Ureaplasma parvum, and Ureaplasma urealyticum organisms in genitourinary samples by PCR-microtiter plate hybridization assay," Journal of Clinical Microbiology, vol. 41, no. 5, pp. 1850-1855, 2003.

[12] R. Safarkar, J. F. Mehrabadi, Z. Noormohammadi, and R. Mirnejad, "Development a rapid and accurate multiplex real time PCR method for the detection Chlamydia trachomatis and Mycoplasma hominis," Journal of Clinical Laboratory Analysis, vol. 31, no. 6, p. e22126, 2017.

[13] K. B. Waites, Y. Rikihisa, and D. Taylor-Robinson, "Mycoplasma and Ureaplasma," in Manual of Clinical Microbiology, American Society for Microbiology, Washington, DC, USA, 2003.

[14] W. M. McCormack, "Susceptibility of mycoplasmas to antimicrobial agents: clinical implications," Clinical Infectious Diseases, vol. 17, no. 1, pp. S200-S201, 1993.

[15] K. B. Waites, R. L. Schelonka, L. Xiao, P. L. Grigsby, and M. J. Novy, "Congenital and opportunistic infections: Ureaplasma species and Mycoplasma hominis," Seminars in Fetal and Neonatal Medicine, vol. 14, no. 4, pp. 190-199, 2009.

[16] E. P. Weledji and M. N. Ngowe, "The challenge of intraabdominal sepsis," International Journal of Surgery, vol. 11, no. 4, pp. 290-295, 2013. 


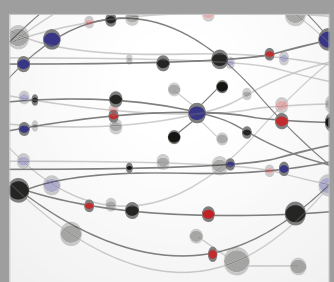

The Scientific World Journal
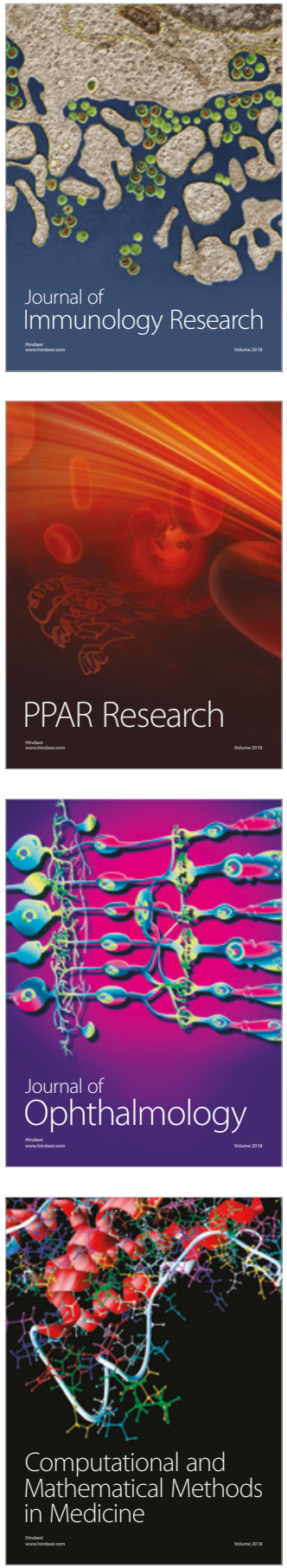

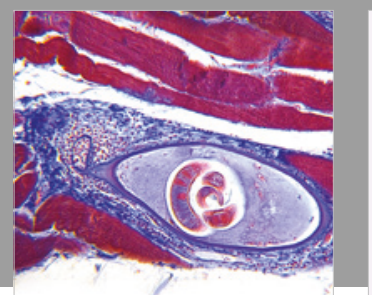

Gastroenterology Research and Practice

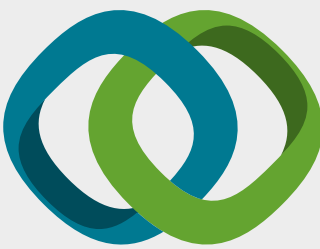

\section{Hindawi}

Submit your manuscripts at

www.hindawi.com
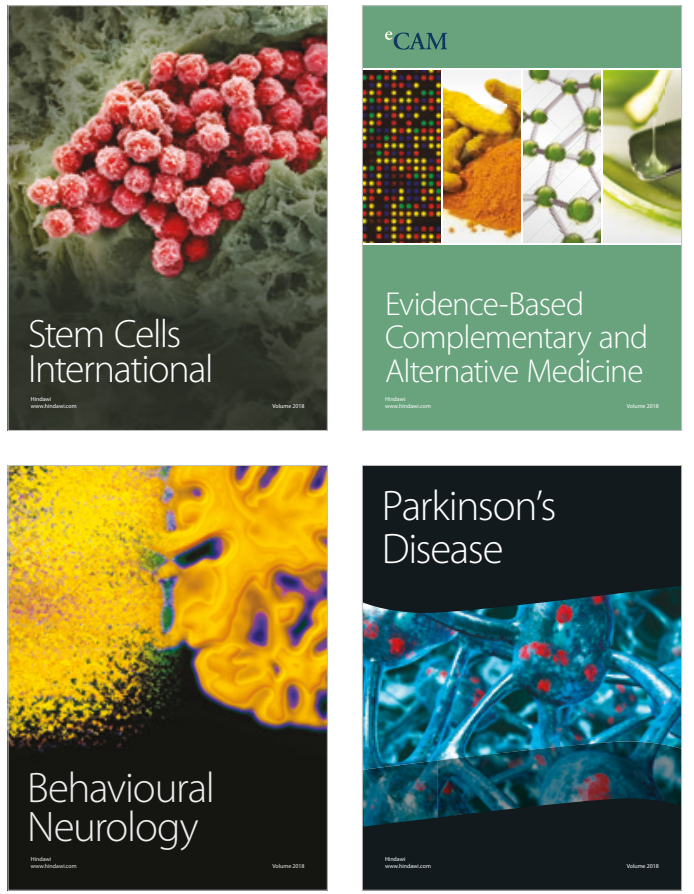

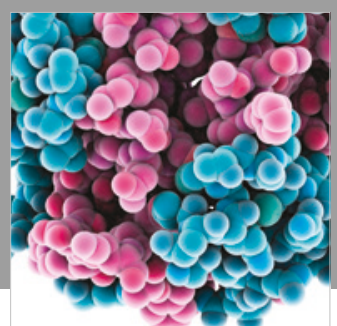

ournal of

Diabetes Research

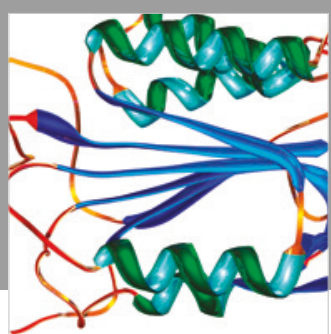

Disease Markers
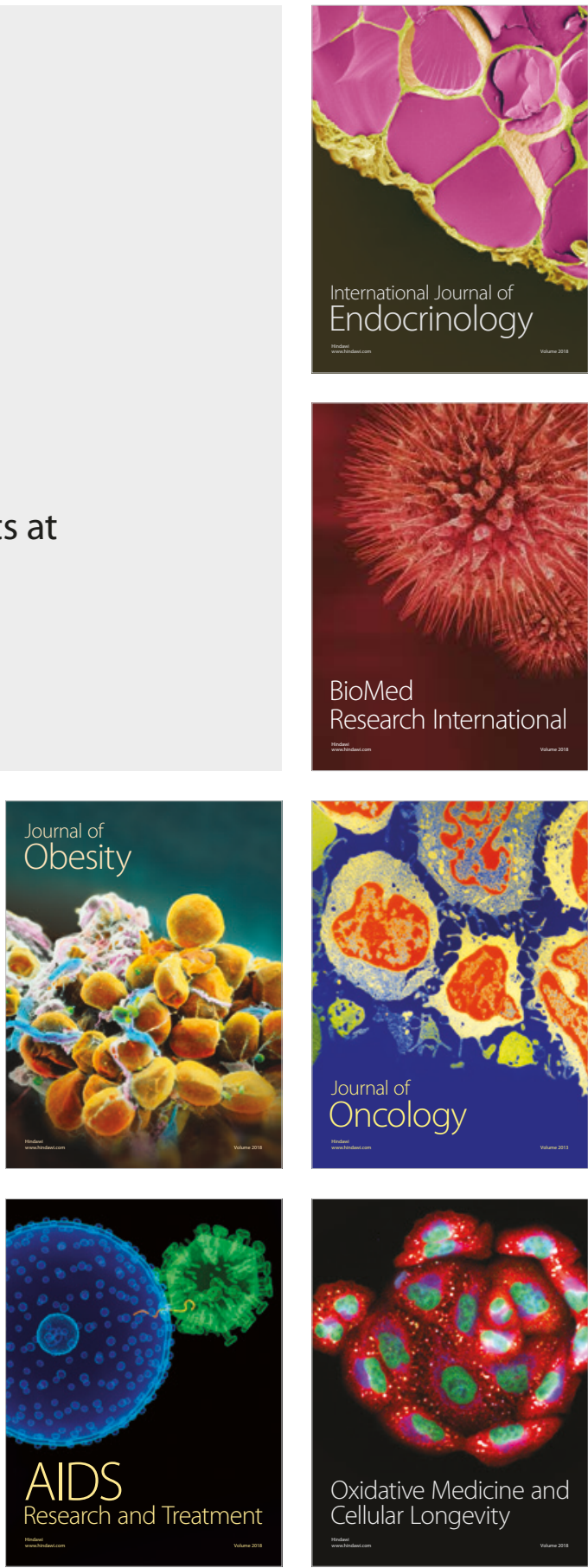